\title{
High Efficiency Electroluminescence from New Blue-Emitting Oligoquinolines Bearing Pyrenyl or Triphenyl Endgroups
}

Jessica M. Hancock, Angela P. Gifford, Christopher J. Tonzola, and Samson A. Jenekhe*

Department of Chemical Engineering and Department of Chemistry, University of Washington, Seattle, Washington 98195-1750

\section{Supporting Information}

Figure S1. Second heating DSC thermograms of BPYPQ (a) and B3PPQ (b) at a heating rate of $10{ }^{\circ} \mathrm{C} / \mathrm{min}$ in nitrogen.

Figure S2. Photoluminescence (PL) decay curves of the PL emission peak of BPYPQ in $10^{-6} \mathrm{M}$ chloroform solution (a) and as a thin film (b) with $381 \mathrm{~nm}$ excitation. Open symbols represent the actual data and the solid lines are fits to the data. Weighted residuals for the single-exponential fit to the solution PL decay curve (c) and for the biexponential fit to the thin film PL decay curve (d).

Figure S3. (a) Photoluminescence (PL) decay curve of the PL emission peak of B3PPQ with $381 \mathrm{~nm}$ excitation. Open symbols represent the actual data and the solid line is fit to the data. (b) Weighted residuals for the biexponential fit to the thin film PL decay curve. 

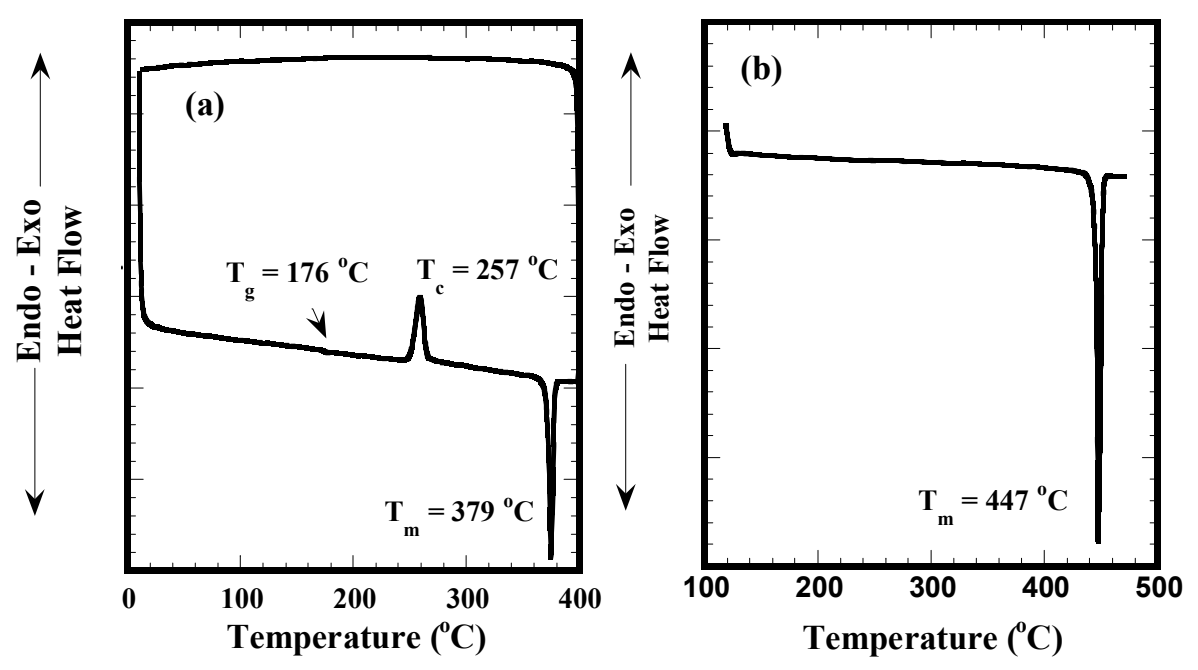

Figure S1. Second heating DSC thermograms of BPYPQ (a) and B3PPQ (b) at a heating rate of $10{ }^{\circ} \mathrm{C} / \mathrm{min}$ in nitrogen. 

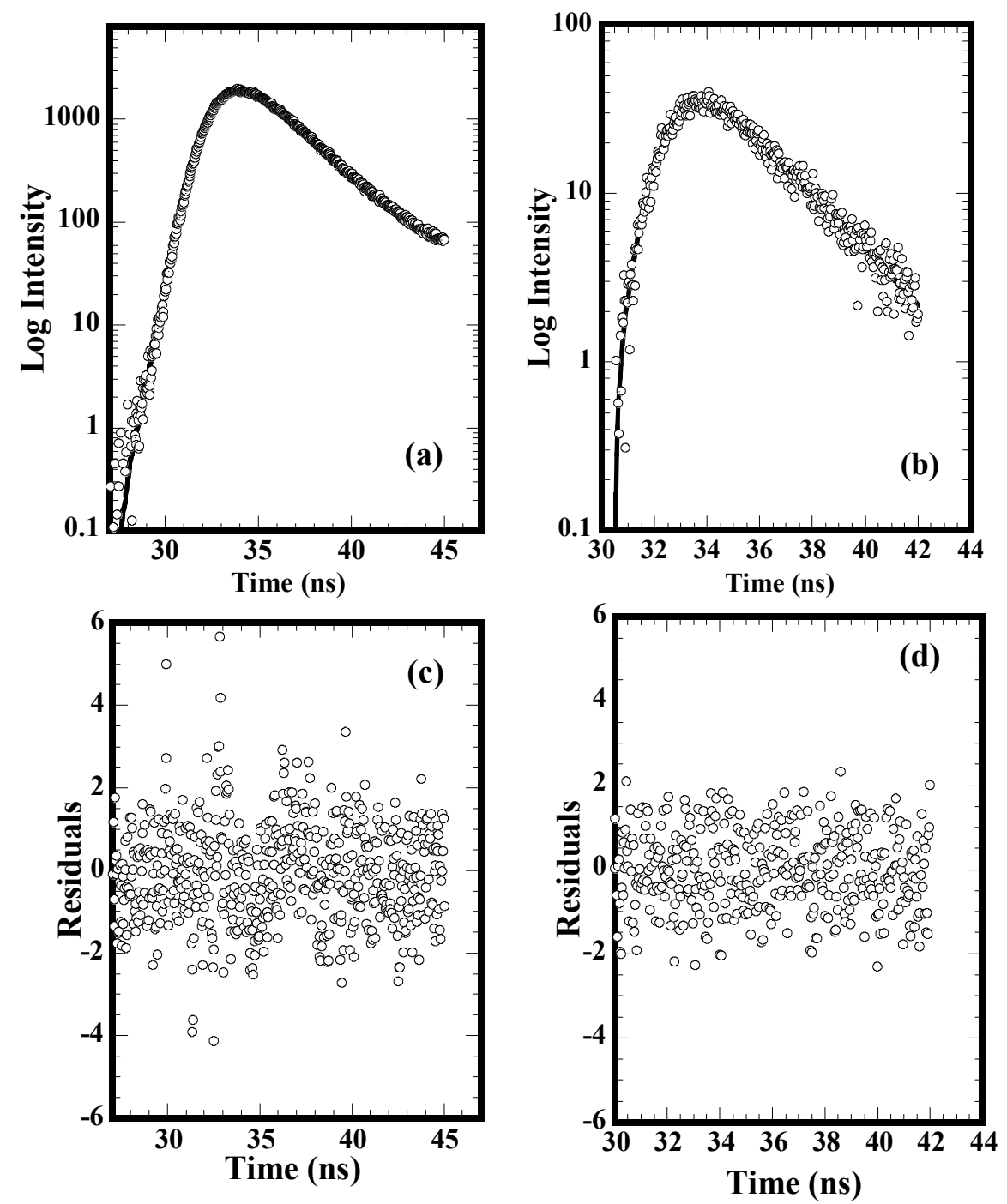

Figure S2. Photoluminescence (PL) decay curves of the PL emission peak of BPYPQ in $10^{-6} \mathrm{M}$ chloroform solution (a) and as a thin film (b) with $381 \mathrm{~nm}$ excitation. Open symbols represent the actual data and the solid lines are fits to the data. Weighted residuals for the single-exponential fit to the solution PL decay curve (c) and for the biexponential fit to the thin film PL decay curve (d). 

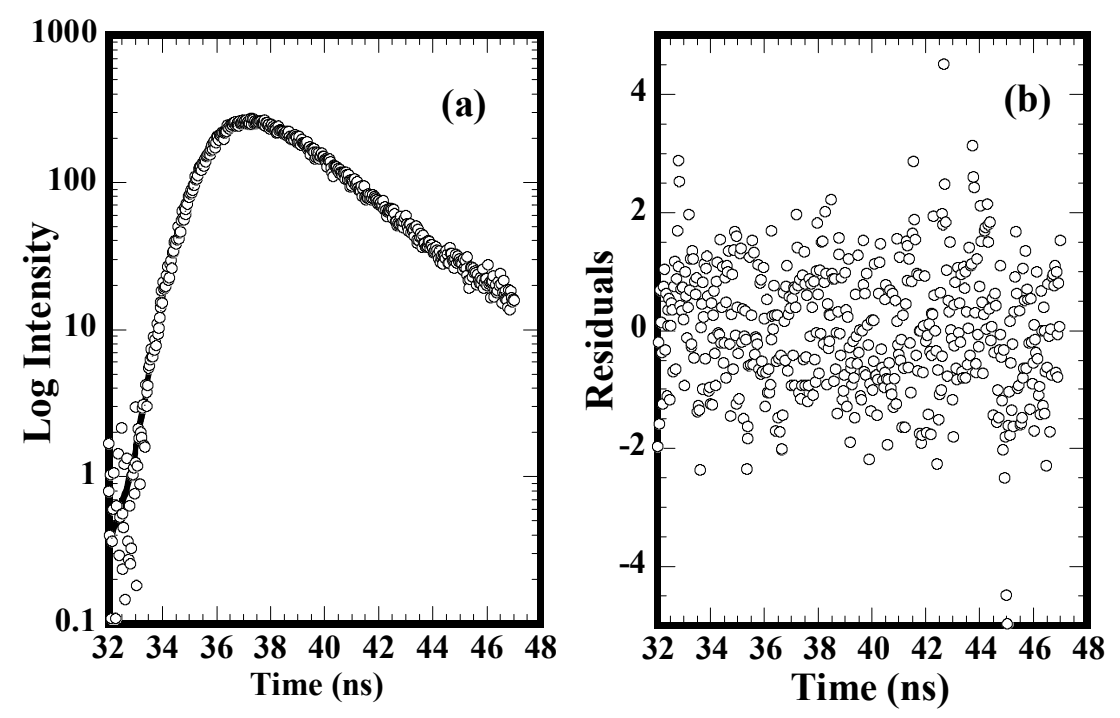

Figure S3. (a) Photoluminescence (PL) decay curve of the PL emission peak of B3PPQ with $381 \mathrm{~nm}$ excitation. Open symbols represent the actual data and the solid line is fit to the data. (b) Weighted residuals for the biexponential fit to the thin film PL decay curve. 